\title{
Measurement of contact potential difference and material distribution by using an SEFM
}

\author{
Keiichiro HOSOBUCHI*, Zhigang JIA*, So ITO*, Yuki SHIMIZU* and Wei GAO* \\ * Nano-Metrology and Control Laboratory, Department of Nanomechanics, Tohoku University, \\ 6-6-01 Aramaki Aza Aoba, Aoba-ku, Sendai 980-8579, Japan \\ E-mail: so.ito@nano.mech.tohoku.ac.jp
}

Received 3 March 2014

\begin{abstract}
This paper presents a method for the measurement of the contact potential difference (CPD) and material distribution on the sample surface by using a scanning electrostatic force microscope (SEFM). In SEFM, the surface profile is evaluated by applying an electrostatic force generated between the probe tip and the sample surface. The amount of electrostatic force relies on both the surface profile and the surface material distribution. To selectively extract the quantitative surface profile, the calculation method to cancel the effect of surface material distribution towards detected electrostatic force is applied in the profile measurement using SEFM. On the other hand, by utilizing the variation of electrostatic force affected by the material distribution, and applying the quantitative profile measurement principle, CPD measurement can be realized. Since the intensity of electrostatic force generated between the probe and the sample surface will rely on the differences of the CPD, the CPD can be calculated by using the frequency shifts of the probe oscillation when two different bias voltages are applied between the tip and the sample. In this paper, firstly, the effect of the surface material distribution on the profile measurement result is reported. The profile measurement result of a sample which consists of two or more materials showed a similar profile with the measurement result obtained by a commercial AFM, which demonstrates that the proposed principle is effective in quantitative measurement of surface profile. Then, the detection sensitivity of the CPD corresponding to the experimental conditions has been confirmed by simulation. In addition, the basic characteristic of the CPD measurement system has been evaluated.
\end{abstract}

Key words : Scanning electrostatic force microscope, Electrostatic force, Noncontact measurement, Contact potential difference, Nano metrology

\section{Introduction}

Scanning probe microscopes (SPMs) represented by scanning tunneling microscope (STM) and atomic force microscope (AFM), have been applied to measure various surface conditions of the device. In addition to the surface observation, SPMs have been conventionally used to measure not only the surface topography but also the surface charges, the material distributions, magnetic characteristic, and many other physical properties (Melitz, et al., 2011, Such, et al., 2004, Hosaka, et al., 1992). The distribution of the physical properties in the surface of the functional devices will cause great influence on the reliability of the device, and thus it has been required to evaluate the local distribution of the surface physical properties with nanometer scale accuracy. The surface physical properties such as the composition of the surface and the local distribution of surface contamination are important parameters for the characterization of the materials.

SPM has many advantages in precision measurement of these devices, such as noncontact measurement, high spatial resolution, and the possibility of measurement in air atmosphere. In particular, the ability of mapping the local distribution of the physical properties will allow the applications to the fields from surface science to the development of new electric devices. On the other hand, there is a trade-off for SPM measurement systems. In conventional SPMs, the local interactions such as the tunneling current of a STM and the atomic force of an AFM can be utilized to control the probe-to-sample within nanometric scale in non-contact condition. In order to detect the local interaction efficiently, it is necessary to maintain the probe-to-sample distance less than $10 \mathrm{~nm}$ during the measurement (Barth, et al., 2011). This small distance causes a risk for collision between the probe tip and the sample owing to the feedback response limitation. Efforts have been made to improve the stability of SPMs, such as increasing the feedback response or optimizing the scanner structure (Ando, et al., 2012), although the scan range and the sample flatness are often limited 

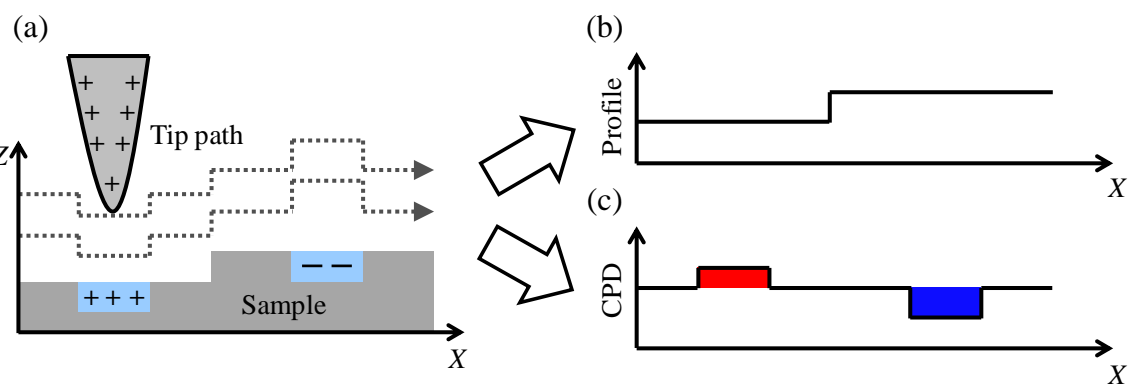

Fig. 1 Quantitative evaluation of surface profile by dual height method and its application towards CPD measurement.

and it is still difficult to realize an SPM without collision. A further improvement of measurement stability for a faster scanning is desired to expand the application of the SPM measurement.

In our previous research, an SEFM has been developed for surface profile measurement in noncontact condition (Gao, et al., 2012). In this new type of SEFM, the surface profile is measured by utilizing the electrostatic force generated between probe tip and the sample surface, which is called the dual height method. Since the electrostatic force is detectable in a large distance, the tip-to-sample distance can be extracted over $200 \mathrm{~nm}$. The large tip-to-sample distance is effective to avoid the collision of the probe tip and the measuring surface. However, the electrostatic force will be affected not only by the surface profile but also the surface material, the tracking path of the probe tip will not always agree with the surface profile (Fig. 1(a)). Therefore, a method for measuring the surface profile by cancelling the influence of material distribution included in the electrostatic force has been proposed. In the proposed method, the electrostatic force is detected at two different tip heights towards the sample surface to calculate the absolute tip-to-sample distance as well as the quantitative surface profile (Fig. 1(b)).

On the other hand, by utilizing the variation of electrostatic force whose intensity depends on the contact potential difference (CPD) between the different materials of the probe and the sample surface, the SEFM can be applied for the measurement of CPD on the sample surface. By utilizing the detected electrostatic force which is affected by both the surface profile and the surface material distribution (Fig. 1(a)), and the calculated quantitative profile (Fig. 1(b)), the CPD over the sample surface can be calculated by cancelling the influence of the surface profile (Fig. 1(c)). Unlike the conventional SPMs, the proposed SEFM has an advantage that the CPD can be measured by maintaining a large tip-to-sample separation during the measurement, and thus it has a possibility to realize crash-free and fast measurement. It will be possible to estimate the types of materials over the sample in noncontact condition since the distribution of the CPD can be detected by modulating bias voltage between the tip and the sample. The principle of measuring the CPD can be applied in the fields of semiconductor fabrication and micro-electro mechanical systems.

In this paper, the influence of the material on the detected electrostatic force is investigated. The calculated profile is evaluated by profile measurement experiment of sample which consists of different materials. Then, the characteristic of the proposed CPD measurement method is evaluated by simulation. Also, an experimental result to evaluate the basic characteristic of the proposed CPD measurement principle by utilizing the developed prototype microscope is presented.

\section{Principle}

In order to obtain the CPD value, it is necessary to measure the tip-to-sample distance at first. The principle of tip-to-sample distance measurement is shown in Fig. 2. As shown in Fig. 2(a), an electrostatic force $F$ is generated by the bias voltage $V_{\text {bias }}$ applied between the probe tip and the sample, and the intensity of the force can be expressed by equation (1) (Belaidi, et al., 1997).

$$
F=\frac{1}{2} \pi \varepsilon_{0} \varepsilon_{r}\left(V_{\text {bias }}+V_{c p d}\right)^{2} \frac{R}{h}
$$

where, $R$ is the tip radius, $h$ is the tip-to-sample distance, $\varepsilon_{0}$ is the dielectric constant of vacuum, $\varepsilon_{r}$ is the dielectric constant of air, and $V_{C P D}$ is the contact potential difference between tip and the sample. As shown in Fig. 2(b), the tip is mounted on a quartz tuning fork (QTF) to detect the electrostatic force by using the piezoelectric effect. The force detected by the probe tip will cause the shift $\Delta f$ from the resonant frequency of the QTF. The value of $\Delta f$ can be expressed as; 


$$
\Delta f=\frac{1}{2} \frac{f}{k} \frac{\partial F}{\partial h}=-\frac{1}{2} \frac{f}{k} \pi \varepsilon_{0} \varepsilon_{r}\left(V_{\text {bias }}+V_{c p d}\right)^{2} \frac{R}{h^{2}}
$$

where, $k$ is the spring constant of the QTF. On the other hand, the frequency shift $\Delta f_{w}$ detected in a tip-to-sample distance of $h+w$ can be expressed by equation (3).

$$
\Delta f_{w}=-\frac{1}{2} \frac{f}{k} \pi \varepsilon_{0} \varepsilon_{r}\left(V_{b i a s}+V_{c p d}\right)^{2} \frac{R}{(h+w)^{2}}
$$

By utilizing equations (2) and (3), the absolute tip-to-sample distance $h$ can be calculated by equation (4).

$$
h=w \frac{\sqrt{\Delta f_{w} / \Delta f}}{1-\sqrt{\Delta f_{w} / \Delta f}}
$$

The CPD which is included in equations (1) to (3) is a value which is defined by the difference of work function of the probe tip and the sample material. The work function is the energy difference between the Fermi energy and the vacuum level. Its value will be varied by the surface condition of the surface such as the types of materials or oxidation conditions. Therefore, it is possible to estimate the local material distribution by monitoring the value of CPD in each measurement points over the sample. The value of the CPD can be calculated by equation (5) (Nonnenmacher, et al., 1991).

$$
\Delta V_{A B}=\phi_{A}-\phi_{B}=\left(w_{A}-w_{B}\right) / e
$$

where $\Phi_{A}$ and $\Phi_{B}$ express the Fermi energy of the material A and $\mathrm{B}, w_{A}$ and $w_{B}$ are the work functions of the materials, and $e$ is the elementary charge. For example, the work functions of Gold, Chrome, and Tungsten have been previously measured to be $5.10 \mathrm{eV}, 4.50 \mathrm{eV}, 4.55 \mathrm{eV}$, respectively (Herbert, et al., 1977). When the sample surface material is Au

(a)

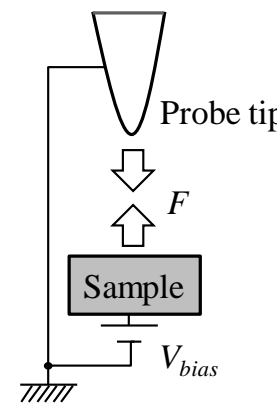

(b)

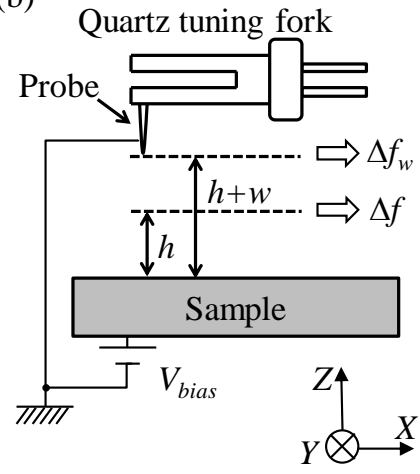

Fig. 2 Measurement principle of tip-to-sample distance.

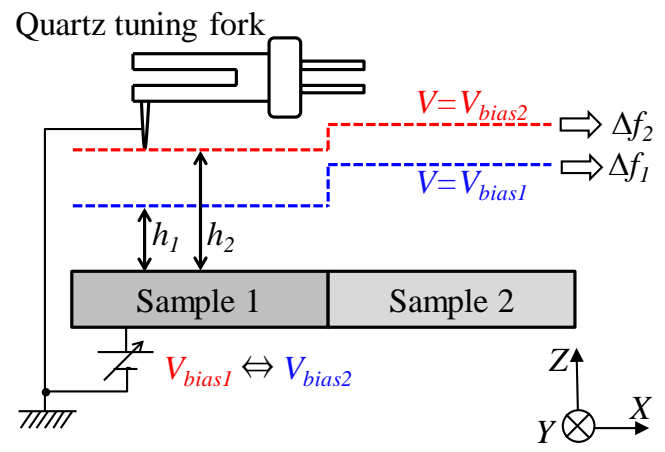

Fig. 3 Measurement principle of CPD. 
and the probe tip material is $\mathrm{W}$, The $\mathrm{CPD}$ of $\mathrm{Au}$ and $\mathrm{W}$ can be calculated to be $-202.3 \mathrm{mV}$ from equation (5). The frequency shift of the QTF will shift accordingly to this value in addition to the applied bias voltage.

The principle of measuring CPD is shown in Fig. 3. First, the bias voltage between the tip and the sample is set to $V_{\text {biasl }}$ and the tip-to-sample distance $h_{l}$ will be calculated by utilizing equation (4). Then, the bias voltage is changed to $V_{\text {bias } 2}$ and the tip-to-sample distance $h_{2}$ will be calculated in the same way. The detected frequency shifts $\Delta f_{1}$ and $\Delta f_{2}$ corresponding to the applied bias voltage $V_{\text {bias } 1}$ and $V_{\text {bias2 }}$ can be expressed by equations (6) and (7), respectively.

$$
\begin{aligned}
& \Delta f_{1}=-\frac{1}{2} \frac{f}{k} \pi \varepsilon_{0} \varepsilon_{r}\left(V_{b i a s 1}+V_{c p d}\right)^{2} \frac{R}{h_{1}^{2}} \\
& \Delta f_{2}=-\frac{1}{2} \frac{f}{k} \pi \varepsilon_{0} \varepsilon_{r}\left(V_{b i a s 2}+V_{c p d}\right)^{2} \frac{R}{h_{2}^{2}}
\end{aligned}
$$

By utilizing equations (6) and (7), the CPD can be calculated by equation (8).

$$
V_{c p d}=\frac{h_{1} \cdot \sqrt{\Delta f_{1}} \cdot V_{b i a s 2}-h_{2} \cdot \sqrt{\Delta f_{2}} \cdot V_{b i a s 1}}{h_{2} \cdot \sqrt{\Delta f_{2}}-h_{1} \cdot \sqrt{\Delta f_{1}}}
$$

In the constant height mode, the tip-to-sample distance $h$ can be cancelled. Equation (9) can then be simplified as;

$$
V_{c p d}=\frac{\sqrt{\Delta f_{1}} \cdot V_{\text {bias } 2}-\sqrt{\Delta f_{2}} \cdot V_{\text {bias } 1}}{\sqrt{\Delta f_{2}}-\sqrt{\Delta f_{1}}}
$$

In the constant height mode, the measurement of the tip-to-sample distance is not necessary, although the distance must be kept constant by applying the open loop scanning. In the open loop scanning, the probe tip position with respect to the sample surface will be settled based on the tracking path of the probe recorded by closed loop scanning in advance. However, in this case the position of the probe tip with respect to the sample surface will be easily affected by the thermal drift. This will cause measurement errors to the calculated CPD value. Therefore, in the actual experiment, the method of applying equation (8) is employed which utilizes the tip-to-sample distance calculated based on the tracking path and the frequency shift detected by the closed loop scanning.

\section{Simulations}

The expected frequency shifts detected in different materials were estimated by simulation. Table 1 shows the example of measurement condition and parameters used in this simulation while the CPD was calculated from the detected frequency shift of QTF. The frequency shift was calculated by using the values of work functions of different materials versus tungsten (W), by using equation (6). The result is shown in Fig. 4. The horizontal axis is the CPD between each material and the $\mathrm{W}$ tip. The vertical axis is the estimated frequency shift using equation (6). As shown in this result, the frequency shift varied for different materials. The difference of the frequency shift between different materials was calculated to be less than $5 \mathrm{mHz}$ in this measurement condition.

Table 1 Example of the experimental condition.

\begin{tabular}{|l|l|l|}
\hline \multicolumn{1}{|c|}{ Parameters } & \multicolumn{1}{c|}{ Value } & \multicolumn{1}{c|}{ Credit } \\
\hline Resonant frequency $f_{\text {reso }}$ & 25000 & $\mathrm{~Hz}$ \\
\hline Spring constant of QTF $k$ & 12900 & $\mathrm{~N} / \mathrm{m}$ \\
\hline Bias voltage $V_{\text {bias }}$ & $-70 \sim 0$ & $\mathrm{~V}$ \\
\hline Contact potential difference $V_{C P D}$ & 0.202 & $\mathrm{~V}$ \\
\hline Tip radius $R$ & 100 & $\mathrm{~nm}$ \\
\hline Tip-to-sample distance $h$ & $40 \sim 300$ & $\mathrm{~nm}$ \\
\hline
\end{tabular}




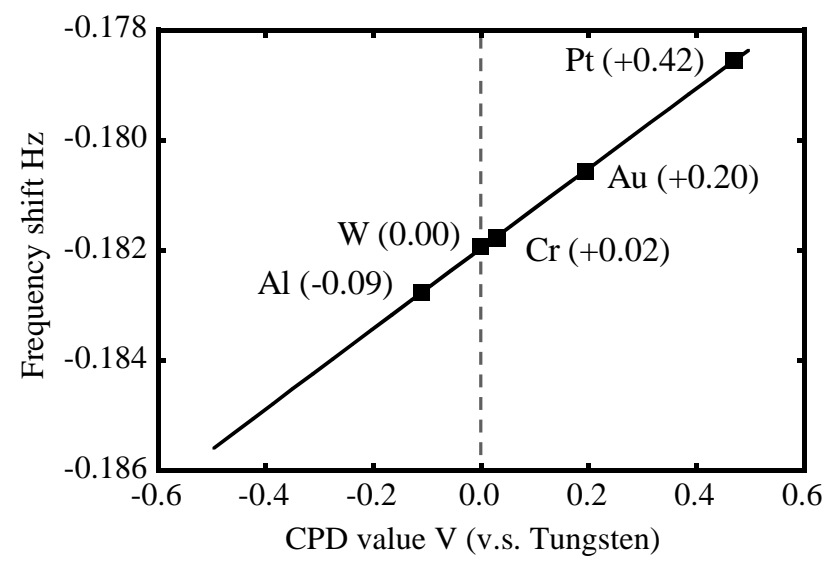

Fig. 4 Simulation result of the relationship between CPD value (towards Tungsten) and frequency shift in different materials.

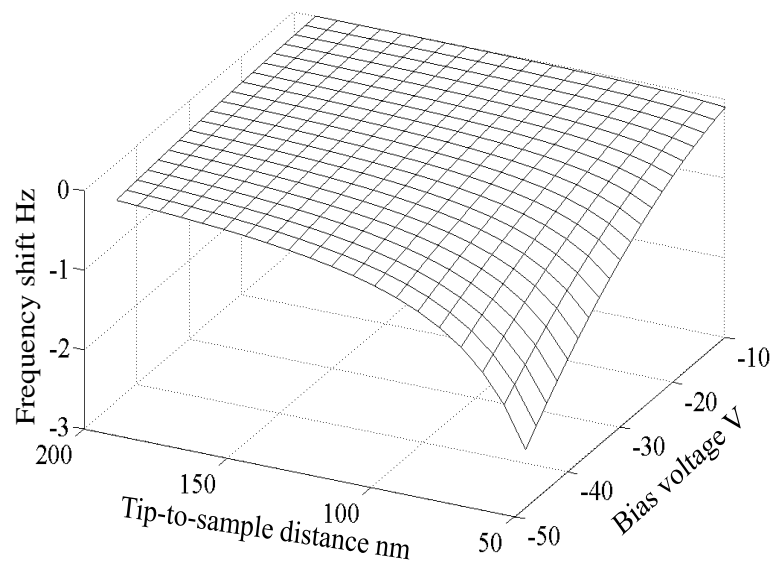

Fig. 5 Simulation result of frequency shift value in different tip-to-sample distance and bias voltage.

The sensitivity of the detection of CPD will be greatly influenced by the bias voltage and the actual tip-to-sample distance. In order to confirm the influence of measurement condition on the frequency shift, the parameters included in equation (6) were changed and the frequency shifts were compared by simulation. The result is shown in Fig. 5. The horizontal axes are the tip-to-sample distance and bias voltage, respectively. The vertical axis indicates the frequency shift. The simulation result shows that the sensitivity gradient is a function of the distance and the bias voltage. With the increase of the bias voltage and the decrease in the tip-to-sample distance, the frequency shift would increase in negative.

It should be noted that a discharge between the tip and the sample will occur when the electric field is larger than 1.0 $\mathrm{V} / \mathrm{nm}$ (Kumar, et al., 2007). For example, when the bias voltage is $-50 \mathrm{~V}$, the tip-to-sample distance should be set larger than $50 \mathrm{~nm}$ in a practical sense.

\section{Experiments}

\subsection{Schematics of the measurement system}

Schematic of the measuring system is shown in Fig. 6. A sharpened tungsten wire was used as the probe to detect the electrostatic force. A Tungsten wire was electrochemically etched and the probe tip radius was sharpened to be about $100 \mathrm{~nm}$. The probe was mounted on the QTF by conductive epoxy. The QTF was oscillated in its resonant frequency by a self-oscillation circuit. When the probe detected the electrostatic force from the sample, the resonant frequency of the QTF was shifted according to the amount of the force. The value of the frequency shift was detected by a phase-locked-loop circuit (PLL), and recorded by the PC. Also, the frequency shift was kept constant by controlling the $\mathrm{Z}$ scanner displacement using a PI controller. When the bias voltage was constant, the electric potential would change due to the distance. In order to maintain the tip-to-sample distance constant, the frequency shift was controlled to be constant. The resolution of the $\mathrm{Z}$ scanner with linear encoder was $0.5 \mathrm{~nm}$, and the nonlinearity was less than $10 \mathrm{~nm}$ over a stroke of $50 \mu \mathrm{m}$ (Goto, et al., 2011). The displacement of the Z scanner was detected by a linear encoder, and the value was recorded by the PC. 
The bias voltage was controlled by a D/A converter, and applied between the probe tip and the sample surface via an amplifier with a magnification of 15 . The electrode of the probe tip was connected to the operational amplifier which was used as the $I-V$ converter, and by the virtual short of the operational amplifier, the probe tip electrical potential would be kept Zero. Therefore, the potential difference between the probe tip and the sample surface would coincide with the output of the bias voltage amplifier.

\subsection{Influence of the surface material on the profile measurement}

In order to confirm the principle of surface profile measurement with the cancellation of surface material distribution affect, the influence of the surface material distribution on the profile measurement result was verified by a profile measurement experiment on a grating shaped sample whose surface was consisted of $\mathrm{Au}$ and $\mathrm{Cr}$ materials. The sample was manufactured by wet etching. The designed pitch and amplitude of the Au/Cr grating were $20 \mu \mathrm{m}$, and 100 nm, respectively.

At first, in order to confirm the surface profile of the sample, the surface profile was measured by a commercial white light interferometer and a commercial dynamic force AFM. The results are shown in Fig. 7 and Fig. 8, respectively. From the results, the pitch of the grating was evaluated to be $20 \mu \mathrm{m}$ which agreed with the designed value, although the amplitude was evaluated to be $80 \mathrm{~nm}$ by the white light interferometer and $110 \mathrm{~nm}$ by the dynamic force AFM. Since the measurement result of amplitude of another step-height sample consisting of monolithic material was

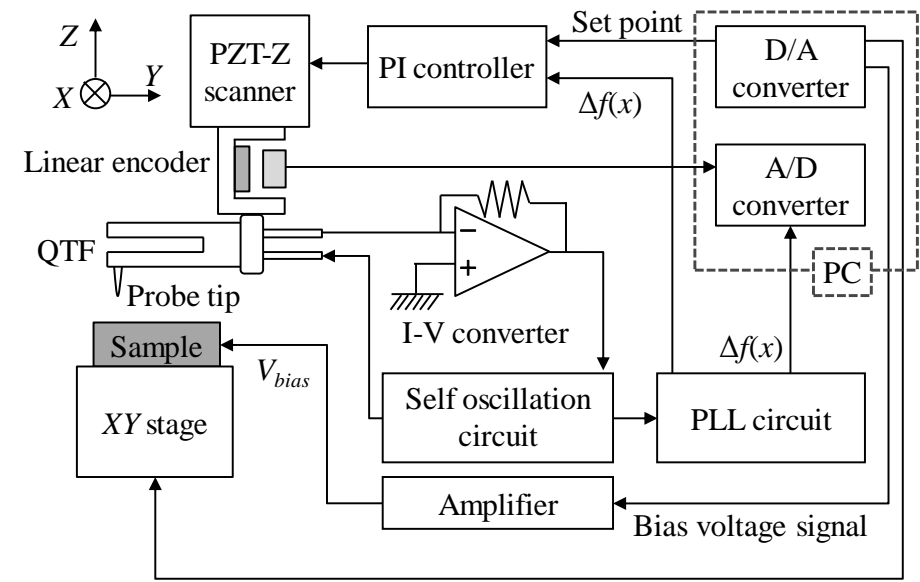

Fig. 6 Schematics of the measurement system.

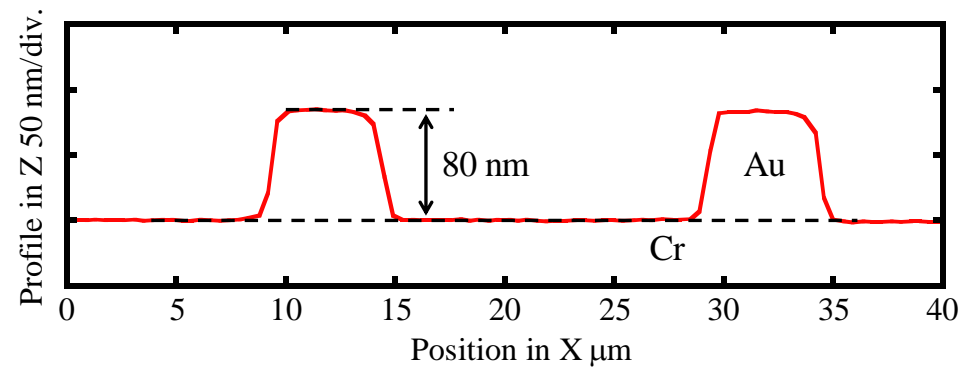

Fig. 7 Section profile of $\mathrm{Au} / \mathrm{Cr}$ gratings measured by conventional white light interferometer.

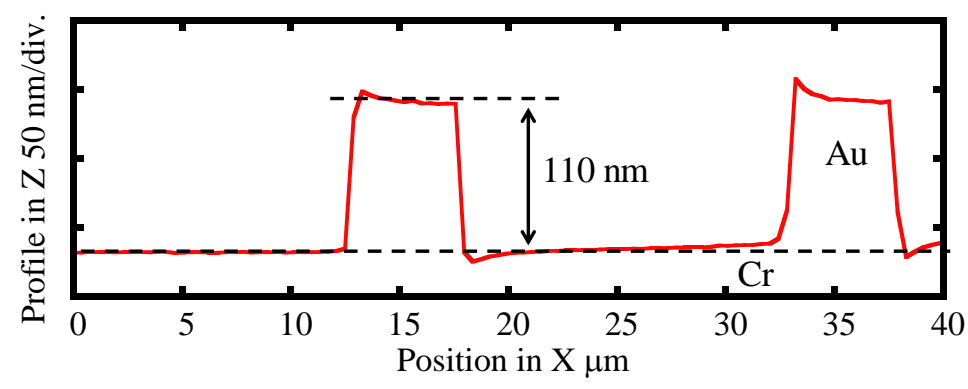

Fig. 8 Section profile of $\mathrm{Au} / \mathrm{Cr}$ gratings measured by conventional dynamic mode AFM. 
evaluated to be the same by the white light interferometer and the dynamic force AFM, it was evident that the variety of the reflective light intensity due to the material distribution caused measurement error to the profile measurement result by the white light interferometer. Since the probe tip intermittently contacted with the sample surface during the measurement in the dynamic force AFM, the influence of the material on the measurement result could be considered to be in the order of sub-nanometer. Therefore, the actual amplitude of the Au/Cr sample could be considered to be 110 nm.

Profile measurement experiments of the $\mathrm{Au} / \mathrm{Cr}$ grating sample were conducted based on the dual height method shown in equation (4). The $Q$ value is a dimensionless parameter that describes the vibration conditions of the resonator. The $Q$ value is defined as $Q=f_{0}\left|f_{l}-f_{2}\right|$, where $f_{0}$ is the resonance frequency of the QTF, $f_{1}$ and $f_{2}$ express the frequency at which the vibrating energy equals the half vibrating energy at the resonance frequency $f_{0}$. As for the probe oscillation of the SPMs, the sensitivity of the interaction force is influenced by the $Q$ value (Morville, et al., 2005). The $Q$ value of the QTF was evaluated to be over 7000. In the experiment, the probe tip position and the frequency shift were detected in two different heights with respect to the sample surface. The sampling frequency was set to be $200 \mathrm{~Hz}$, and the average value of 100 data points per one measurement point was defined as the measurement value of single measurement point. After the measurement of one measurement point, the probe tip was transferred along the $X$ axis to the next point. By repeating this procedure, one line profile measurement was conducted. The scanning range was set to be $30 \mu \mathrm{m}$, and the total measurement time was $300 \mathrm{~s}$.

The results are shown in Figs. 9, and 10. $d(x)$ and $d_{w}(x)$ were the tracking path detected in two different height positions. $h(x)$ was the calculated tip-to-sample distance, and $\mathrm{s}(\mathrm{x})$ was the profile. Here, the profile was calculated by subtracting the tip-to-sample distance from the tracking path of probe tip, which could be expressed as $s(x)=d(x)$ - $h(x)$. Firstly, the amplitudes of the tracking paths $d(x)$ and $d_{w}(x)$ were detected to be both $100 \mathrm{~nm}$, which was smaller compared to the profile measurement result by the commercial AFM. On the other hand, the calculated tip-to-sample distance had a difference of $10 \mathrm{~nm}$ between the higher and lower points of the grating. As a result, the amplitude of the surface profile was calculated to be $110 \mathrm{~nm}$, which agreed with the AFM measurement result. The reason of the difference between the tracking path and the calculated profile was considered to be the affect of surface material

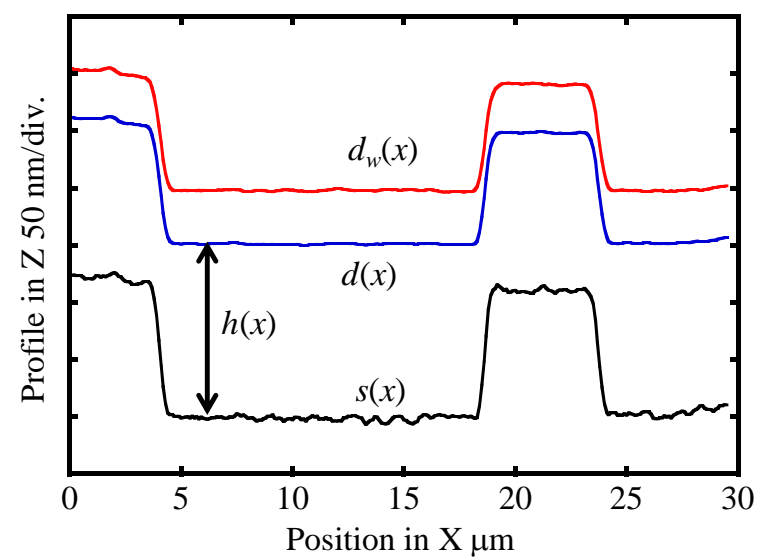

Fig. 9 Tracking path of the probe tip and calculated profile of $\mathrm{Au} / \mathrm{Cr}$ gratings.

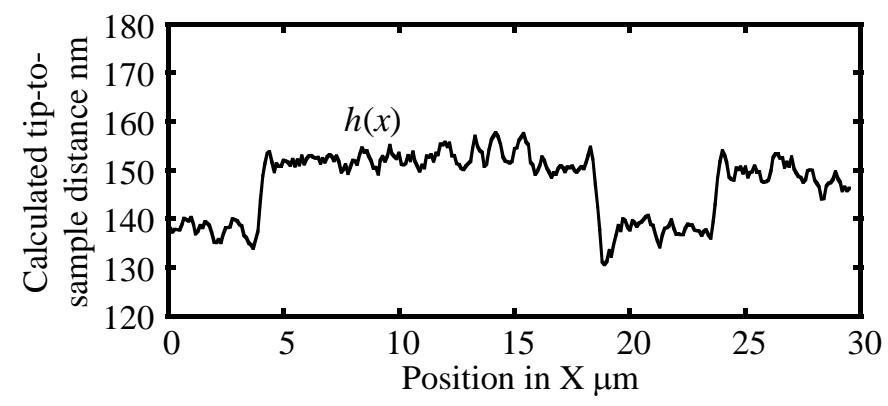

Fig. 10 Calculated tip-to-sample distance. 
distribution. Theoretically, when using a monolithic material sample, the tip-to-sample distance will be kept constant during the scanning. When the probe tip is tracked continuously towards the sample surface, the feedback error will cause change to the tip-to-sample distance. Here the delay of the feedback was considered and the data were obtained in two different height positions in each measurement point so that the feedback delay would not cause any change in the tip-to-sample distance. Moreover, every parameter included in equation (2) which effects the electrostatic force except $V_{C P D}$ will not be changed during the scanning. From these reasons, the difference of the $V_{C P D}$ that was caused by the material distribution appeared in the tracking path, thus the tip-to-sample distance changed between the higher and lower points of the grating. From the above results, it was experimentally confirmed that the surface profile can be effectively measured by using the dual height method, according to which the influence of the CPD distribution on the sample surface can be removed.

\subsection{Measurement of the CPD}

The relationship between the frequency shift of the probe oscillation and the probe-to-sample distance was investigated over two different materials of $\mathrm{Au}$ and $\mathrm{Cr}$. The probe tip was approached towards the sample surface in a constant velocity. The probe tip displacement and the frequency shift were recorded. The sampling frequency was set to $5 \mathrm{kHz}$, and the displacement applied to the probe tip per step was $1 \mathrm{~nm}$. The average value of 50 data points sampled per step was defined as the measurement value. The bias voltage was set to $-50 \mathrm{~V}$. The result is shown in Fig. 11. The horizontal axis is the estimated tip-to-sample distance calculated from equation (6), and the vertical axis is the frequency shift of the probe oscillation. The solid line shows the raw data of the detected frequency shift, and the dotted line shows the fitting curve of the raw data. It can be confirmed from the results that the frequency shift was proportional to the square inverse of the tip-to-sample distance, as is shown in equation (6). The $R^{2}$ values of the inverse fitting curve of each force curves were calculated to be both over 0.97. The results of the two curves had a difference of $0.02 \mathrm{~Hz}$ when the tip-to-sample distance was $200 \mathrm{~nm}$. Also, it can be confirmed that the frequency shift value detected over $\mathrm{Cr}$ tend to be larger than that of $\mathrm{Au}$. This tendency can be explained from the difference of the $\mathrm{CPDs}$ of $\mathrm{Au}$ and $\mathrm{Cr}$ with respect to tungsten, which is shown in Fig. 4.

The CPD values of $\mathrm{Au}$ and $\mathrm{Cr}$ were calculated based on the calculation method shown in equation (8). The timing chart of the measurement procedure is shown in Fig. 12. Two different parameters, the frequency shift set point and the bias voltage, were set as shown in Table 2 . Firstly, the frequency shift set point was set to $\Delta f_{1}$, and the bias voltage was set to $V_{\text {biasl }}$. Next, only the frequency shift set point was changed to $\Delta f_{2}$. From this two conditions, the probe positions could be expressed as $d\left(\Delta f_{1}, V_{\text {biasl }}\right)$ and $d\left(\Delta f_{2}, V_{\text {biasl }}\right)$, respectively, which were used to calculate the tip-to-sample

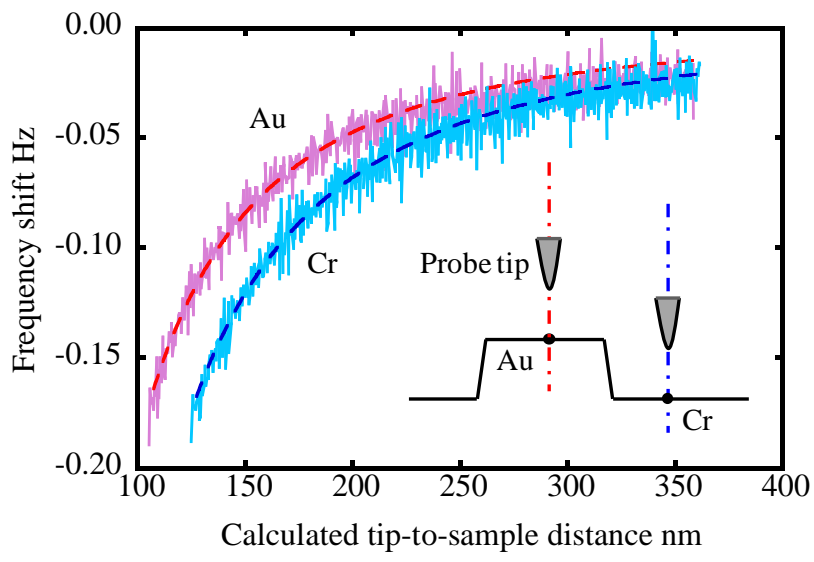

Fig. 11 The relationship between frequency shift and tip-to-sample distance measured at single point over $\mathrm{Au}$ and $\mathrm{Cr}$.

Table 2 Parameters set in the CPD measurement.

\begin{tabular}{|c|c|c|}
\hline Frequency shift set point & Bias voltage & Probe position \\
\hline$\Delta f_{l} \quad(-0.087 \mathrm{~Hz})$ & $V_{\text {biasl }}(45 \mathrm{~V})$ & $d\left(\Delta f_{l}, V_{\text {biasl }}\right)$ \\
\hline$\Delta f_{2} \quad(-0.057 \mathrm{~Hz})$ & $V_{\text {biasl }} \quad(45 \mathrm{~V})$ & $d\left(\Delta f_{2}, V_{\text {biasl }}\right)$ \\
\hline$\Delta f_{2} \quad(-0.057 \mathrm{~Hz})$ & $V_{\text {bias } 2} \quad(55 \mathrm{~V})$ & $d\left(\Delta f_{2}, V_{\text {bias } 2}\right)$ \\
\hline
\end{tabular}



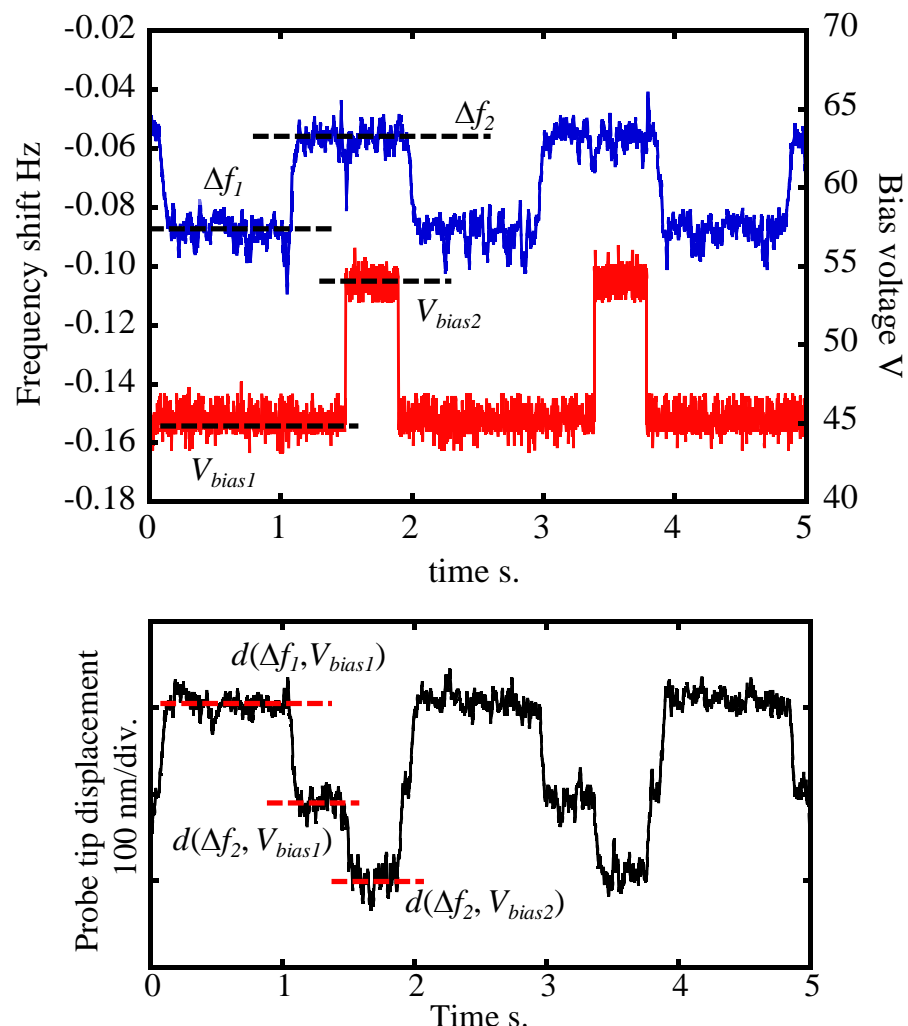

Fig. 12 Timing chart of CPD measurement by vertical reciprocation scanning mode.

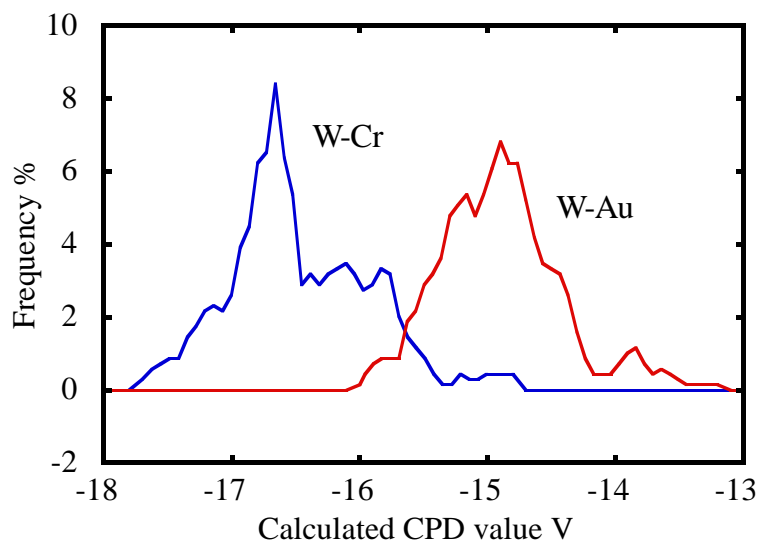

Fig. 13 Histogram of the measured CPD value of $\mathrm{W}-\mathrm{Au}$ and $\mathrm{W}-\mathrm{Cr}$.

distance. Next, by fixing the value of frequency set point $\Delta f_{2}$, the bias voltage was changed to $V_{\text {bias } 2}$. From the second and third states at which the probe positions were $d\left(\Delta f_{2}, V_{\text {biasl }}\right)$ and $d\left(\Delta f_{2}, V_{\text {bias } 2}\right)$, respectively, the CPD value was calculated by using equation (8). This procedure of changing the frequency and the bias voltage can be confirmed in Fig. 12. By repeating this procedure in single point over the $\mathrm{Au}$ and $\mathrm{Cr}$ surfaces, respectively, the CPD values were calculated. The experiment was repeated four times for each material. To confirm the tendency of the measured values, the histogram of the measured CPD values has been calculated for each material. The number of total measurement points for each material was 200 .

The results of the histogram of the measured CPD values are shown in Fig. 13. The horizontal axis shows the CPD value and the vertical axis is the frequency of the data points. The result shows that the distribution of the calculated $\mathrm{CPD}$ values of $\mathrm{Au}$ and $\mathrm{Cr}$ could be distinguished. This indicates that the material distribution could be confirmed from the calculated CPD value. On the other hand, each CPD value had a large difference compared to the experimental value in previous researches (W-Cr: $+18.4 \mathrm{mV}, \mathrm{W}-\mathrm{Au}:+202.3 \mathrm{mV}$ ). Two reasons can be considered for the difference. One is the change of the work function of the sample surface or the probe tip, due to the meniscus layer on the sample surface or the air atmosphere between the probe and the sample surface. Although the work function of the oxide tungsten shows a value more than two times larger compared with that of the pure tungsten, which also causes large 
difference to the CPD value, the influence of the oxidization layer would be limited because of its thin thickness. As shown in the previous research (Kim and Kang, 2011), the measurement value of the CPD is also influenced by thickness of thin layer. On the other hand, thickness of the natural oxidation layer which is generated on the metal or semiconductor surface is less than $10 \mathrm{~nm}$ in general (Mathieu, et al., 1984). Therefore, the influence of the oxidization layer is restrictive and it is estimated that the difference of the CPD is mainly dominated by the difference of the measurement conditions such as temperature and humidity. However, since the difference of the materials on the sample surface can be distinguished by applying the CPD measurement method based on the SEFM, the comparative measurement of CPD over sample surface which consist of known-materials can be realized.

\section{Conclusion}

A method for calculating the CPD value by using an SEFM has been proposed. The effect of the surface material distribution on the profile measurement result has been evaluated. The result showed that the material distribution will cause difference to the detected electrostatic force. The effectiveness of the principle of surface profile measurement using SEFM has been confirmed from the experiment. The sensitivity of CPD detection has been evaluated by simulation. The sensitivity for detecting CPD can be improved when the tip-to-sample distance gets smaller and the bias voltage between the tip and the sample gets larger. Experiments of the CPD measurement have been carried out to demonstrate the feasibility of the CPD calculation method. The relationship between the frequency shift of the QTF and the tip-to-sample distance over two different materials had different values in the order of sub-Hz, which can be explained from the simulation result. The calculated CPD values of the $\mathrm{Au}$ and the $\mathrm{Cr}$ areas have been confirmed to have different value distributions, although the absolute values were different compared to the values calculated from the reference values of work function. The meniscus layer over the sample, oxidization of the probe tip, or the thickness of the metal layer has been considered to be factors of value difference. Improvement of the experimental environment as well as the measurement sample is necessary.

\section{Acknowledgement}

This research is supported by JSPS and Osawa Scientific Studies Grants Foundation.

\section{References}

Ando, T., Uchihashi, T. and Kodera, N., High-speed atomic force microscopy, Japanese journal of applied physics, Vol. 51 (2012), 08KA02.

Barth, C., et al., Recent trends in surface characterization and chemistry with high-resolution scanning force methods, Advanced materials, Vol. 23(4) (2011), pp.477-501.

Belaidi, S., Girard, P. and Leveque, G., Electrostatic forces acting on the tip in atomic force microscopy: Modelization and comparison with analytic expressions, Journal of applied physics, Vol. 81/3 (1997), pp.1023-1030.

Gao, W., et al, A noncontact scanning electrostatic force microscope for surface profile measurement, CIRP annals manufacturing technology, Vol. 61, Issue 1 (2012), pp.471-474.

Goto, S., Hosobuchi K. and Gao, W., An ultra-precision scanning tunneling microscope Z-scanner for surface profile measurement of large amplitude micro-structures, Measurement science and technology, Vol. 22 (2011), 085101.

Herbert B. M., The work function of the elements and its periodicity, Journal of applied physics, Vol. 48, 4729 (1977), 10.1063/1.323539.

Hosaka, S. and Kikukawa, A., Study of magnetic stray field measurement on surface using new force microscope, Japanese journal of applied physics part 2-letters, Vol. 31 (1992), pp.908-911.

Kim, H., et al, Effective work function control of indium-tin-oxide electrodes, Journal of the Korean physical society, Vol. 59(4) (2011), pp. L2655-L2657.

Kumar, R., et al, Understanding Sub-20 nm breakdown behavior of liquid dielectrics, Physical review letters, Vol. 99 (2007), pp.017601.

Mathieu, H., el al., Thickness of nature oxide films determined by AES and XPS with/without sputtering, Journal of vacuum science \& technology A, Vol. 3(2) (1984), pp. 331-335.

Melitz, W., et al., Kelvin probe force microscopy and its application, Surface science reports, Vol. 66(1) (2011), pp.1-27.

Morville, J., et al., Q-factor optimization of a tuning-fork/fiber sensor for shear-force detection, Applied physics letters, Vol. 86, 064103 (2005).

Nonnenmacher, M., O’Boyle, M. P. and Wickramasinghe, H. K., Kelvin probe force microscopy, Applied physics letters, Vol. 58 (25) (1991), pp.2921.

Such, M. W., Kramer, D. E. and Hersam M. C., Reproducible lateral force microscopy measurements for quantitative comparisons of the frictional and chemical properties of nanostructures, Ultramicroscopy, Vol. 99(2-3) (2004), pp.189-196. 